\title{
Gleichheit und Sicherheit als Voraussetzung für Lebensqualität?
}

Was macht Menschen glücklich? Möglichst gleich verteilte Einkommen, so lautet eine für manche provozierende These. Der Beitrag zeigt, dass Gleichheit zwar eine notwendige, aber keine hinreichende Bedingung für ein hohes Niveau an Lebensqualität ist, und dass sie sich nicht auf materielle Gleichheit beschränken kann. Auch das Wohlstandsniveau und der institutionelle Kontext eines Landes sind für die wahrgenommene Lebensqualität des Einzelnen entscheidend. Lebenszufriedenheit ist vor allem dort hoch, wo soziale Sicherung verlässlich ist, wo Chancengleichheit ein selbstbestimmtes Leben ermöglicht und die Bevölkerung der Politik und Regierung vertrauen kann.

\section{Einleitung}

Je ungleicher Gesellschaften sind, desto größer sind ihre sozialen Probleme mit beispielsweise Kriminalität, Kindersterblichkeit, Selbstmordraten, Übergewicht oder verbreitetem Misstrauen. Und je weiter der Abstand zwischen Arm und Reich ist, desto eher leiden auch Gesundheit und Wohlbefinden der Gesamtbevölkerung. So argumentieren Richard Wilkinson und Kate Pickett in ihrem Bestseller „The Spirit Level. Why Equality Is Better for Everyone“ (Wilkinson/Pickett 2009). Trotz Produktivitätszuwächsen und Einkommenssteigerungen gehe es, so die Autoren, den Menschen nicht stetig besser. Sowohl die Lebenserwartung als auch die Lebenszufriedenheit stagnierten, wenn ein gewisser Level an materiellem Wohlstand erreicht sei. Von mehr Gleichheit, so die These, profitierten alle Gesellschaftsmitglieder, nicht nur die Benachteiligten. Die politische Empfehlung lautet somit: Gleichheit statt Wachstum.

Die methodischen Verkürzungen und vereinfachenden Deutungen dieses Buches sollen hier nicht besprochen werden (Saunders 2010; Liebig 2010). Es trifft unbestritten einen Nerv der Zeit und deckt sich mit Bestrebungen, gesellschaftlichen Fortschritt nicht mehr nur anhand des Bruttoinlandsprodukts zu messen, sondern weitere Indikatoren, die Umweltbelastungen, Chancengleichheit und individuelle Lebensqualität zum Inhalt haben, stärker zu berücksichtigen (Stiglitz et al. 2009). Ein Kernthema der Sozialdemokratie - Gleichheit und Gerechtigkeit als Motor einer besseren Gesellschaft - rückt damit ins Zentrum der politischen Aufmerksamkeit.

Die Frage, welchen Einfluss nicht nur ökonomische, sondern auch politische und wohlfahrtsstaatliche Rahmenbedingungen auf die Lebenschancen des Einzelnen haben, ist alles andere als belanglos und hat eine langjährige Forschungstradition in den Sozialwissenschaften. Das historisch-kulturell geprägte Verhältnis zwischen Markt und Staat ist länderspezifisch, bringt einen unterschiedlichen Umgang mit sozialer Ungleichheit zum Ausdruck und offenbart dessen akzeptiertes Ausmaß und dahinterliegende moralische Wertvorstellungen. Dies wiederum entscheidet mit darüber, wann Ungleichheit als Ungerechtigkeit empfunden wird. Weit verbreitetes Ungerechtigkeitsempfinden birgt Konfliktpotenzial: Ein hohes Maß an Ungleichheit bringt Verunsicherung und Ängste mit sich, was den Zusammenhalt und die Integrationsfähigkeit einer Gesellschaft unweigerlich schwächt und Lebenschancen beschneidet.

Ich möchte zwei Thesen von Wilkinson und Pickett zum Anlass für die folgenden Ausführungen nehmen: Zum einen die Verallgemeinerung, dass nicht das Einkommensniveau, sondern ausschließlich die Einkommensungleichheit für die Verbreitung sozialer Problemlagen und eine damit einhergehende schlechte Lebensqualität verantwortlich seien. Und daran anschließend die Behauptung, dass in einem Land mit hoher (Einkommens-) Ungleichheit nicht nur Benachteiligte eine niedrigere Lebensqualität haben, sondern auch ökonomisch und sozial Privilegierte.

Bei den folgenden Ausführungen handelt es sich nicht um eine Überprüfung der Aussagen von Wilkinson und Pickett im Sinne einer Replikation unter anderen me- thodischen Vorzeichen. Vielmehr geht es darum, den behaupteten Zusammenhang zwischen wahrgenommener Lebensqualität (Lebenszufriedenheit) und Einkommensgleichheit zu überprüfen. Zwar sind Qualitätsmerkmale einer Gesellschaft zur Steigerung der Lebensqualität unbestritten wichtig und müssen deshalb in ihrer Bedeutsamkeit stärker in den Mittelpunkt gerückt werden. Es greift aber zu kurz, wenn man sich dabei auf das Postulat materieller Gleichheit beschränkt. Wir werden sehen - und bestätigen damit Befunde bisheriger Forschung -, dass die Lebenszufriedenheit mit dem Ausmaß an Umverteilung in einer Gesellschaft variiert. Aber auch weitere Faktoren wie das absolute Niveau der wirtschaftlichen Produktivität eines Landes und die Funktionsfähigkeit und Vertrauenswürdigkeit einer Demokratie sind für die Lebensqualität ausschlaggebend. Diese wohlfahrtsstaatlichen Rahmenbedingungen hängen eng mit dem Ausmaß an Ungleichheit einer Gesellschaft zusammen, lassen sich aber nicht darauf reduzieren (Esping-Andersen/Myles 2009).

Die Frage nach der allgemeinen Lebenszufriedenheit ist ein Indikator subjektiven Wohlbefindens, der als Summenmaß für Lebensqualität international anerkannt ist (Glatzer/Zapf 1984; Diener et al. 1999; Layard 2005). Lange Zeit verpönt als wissenschaftlich nicht belastbar,

\footnotetext{
Petra Böhnke, Dr. phil., ist Leiterin der Schumpeter-Nachwuchsgruppe "Soziales Kapital im Lebensverlauf" am Wissenschaftszentrum Berlin für Sozialforschung (WZB). Arbeitsschwerpunkte: Armut und soziale Integration, Partizipation im Lebensverlauf, Lebenszufriedenheit im Wohlfahrtsstaat. e-mail: boehnke@wzb.eu
} 
gilt er mittlerweile als ein entscheidendes Kriterium für Erfolg und Misserfolg politischen Handelns. Ich möchte hier die Frage beantworten, ob egalitäre und sichere Gesellschaften eine höhere Lebenszufriedenheit aufweisen als weniger egalitäre und unsichere. Dafür werde ich den Diskussions- und Forschungsstand $\mathrm{zu}$ individuellen und gesellschaftlichen Determinanten von subjektivem Wohlbefinden referieren (Abschnitt 2 und 3) und mit eigenen empirischen Schlaglichtern (Abschnitt 4) untermauern. Das Grundproblem bei der Suche nach der Erklärung von Länderunterschieden im subjektiven Wohlbefinden besteht darin, dass zumeist nur auf Länderebene argumentiert wird, ohne die Verteilung sozio-ökonomischer Merkmale innerhalb der entsprechenden Bevölkerung zu berücksichtigen. Ich möchte deshalb zeigen, welchen eigenständigen Stellenwert die Qualität einer Gesellschaft (z.B. in Form von Sicherheit, Gleichheit, Gerechtigkeit und Wohlstand) für die perzipierte Lebensqualität hat, und prüfen, welche Bedeutung der Gleichheit im Sinne von Einkommensgleichheit dabei zukommt. Letztlich geht es, wie das Fazit dann nochmals hervorhebt (Abschnitt 5), um das Verhältnis individueller Lebensbedingungen und wohlfahrtsstaatlicher Kontexte für das Verständnis subjektiven Wohlbefindens.

\section{Lebensqualität und Gesellschaft}

Unter Lebensqualität kann sich vermutlich jeder intuitiv etwas vorstellen. Die konkreten Assoziationen, theoretischen Ableitungen und Operationalisierungen sind aber sehr unterschiedlich. Dennoch gehört der Begriff mittlerweile europaweit zum Standard-Repertoire sozialpolitischer Positionierungen. In Deutschland hat er sich im Rahmen der Sozialindikatorenund Wohlfahrtsforschung der 1970er Jahre etabliert (Zapf 1972, 1999) und sich in seiner inhaltlichen Ausrichtung an internationalen Weiterentwicklungen der Wohlfahrtsökonomie seit den 1920er Jahren orientiert (z.B. Campbell et al. 1976; Erikson 1974). „Lebensqualität“ steht für eine mehrdimensionale, individuelle und kollektive Vorstellung von Wohlfahrt und bezieht sich auf materielle und immaterielle
Lebensbedingungen sowie auf subjektives Wohlbefinden gleichermaßen. Die für die deutsche Sozialberichterstattung typische Verknüpfung objektiver Lebensbedingungen, subjektiven Wohlbefindens und der Qualität der Gesellschaft für das Verständnis von Lebensqualität lehnt sich an die Arbeiten des Finnen Eric Allardts an, der dafür Dimensionen wie sozialen Rückhalt, Zugehörigkeit und das Bedürfnis nach Sicherheit anmahnte (Allardt 1976, 1993).

Der Grundgedanke für das gelungene Zusammenspiel von Qualität der Gesellschaft (die "gute" Gesellschaft) und individueller Wohlfahrt (das "gute“ Leben) hat eine lange politisch-philosophische Theoriegeschichte und prägt die Kontroverse um die Entwicklung und Ausrichtung sozialstaatlichen Handelns. Bei Thomas H. Marshall findet er sich prägnant formuliert: Wirtschaftliche Wohlfahrt und Sicherheit sind als soziale Grundrechte und deren Garantie als Voraussetzung für die Verringerung sozialer Ungleichheit zu verstehen (Marshall 1992). Frei sein von „elementarer Not und Furcht“, gilt auch bei Dahrendorf (1992, S. 65) als soziales Grundrecht und Voraussetzung zur Schaffung erweiterter Optionen und Lebenschancen der Einzelnen im Sinne sozialer Gerechtigkeit und Chancengleichheit für alle. Soziale Gerechtigkeit wird über unterschiedliche Gerechtigkeitstheorien hinweg (siehe hierzu Kronauer/Schmid in diesem Heft) wesentlich als Gleichheit verstanden. Staatliches Handeln soll maßgeblich darauf abzielen, allen Mitgliedern einer Gesellschaft gleichermaßen ein gutes und sicheres Leben zu ermöglichen.

Die gegenwärtige Stoßrichtung dieser Debatte konzentriert sich weniger auf Verteilungsgerechtigkeit und Ergebnisgleichheit, sondern stellt die Schaffung von Bedingungen für gleiche Startchancen und die ungehinderte Ausschöpfung individueller Potenziale in den Mittelpunkt. Besonders einflussreich für diese Marschrichtung der europäischen Sozialpolitik sind die Ausführungen von Amartya Sen (1999) zum Zusammenspiel von Politik und Lebensqualität: Sein Konzept der Verwirklichungschancen zielt darauf, individuelle Handlungsfreiheit und ihre Abhängigkeit von sozialen, politischen und ökonomischen Rahmenbedingungen gemeinsam in den Blick zu bekommen. Verwirklichungschancen versteht er als Kompetenzen, Fähigkeiten und Handlungsoptionen, die es Menschen ermöglichen, am gesell- schaftlichen Leben teilzuhaben und einen Lebensweg zu wählen, der den Minimalvoraussetzungen eines würdigen Daseins in dem jeweiligen Land entspricht. Sie sind eng an Ressourcen gebunden, gehen aber darüber hinaus. Sen plädiert in Anlehnung an Rawls für ein Verständnis von Grundgütern, das neben materiellem Wohlstand auch Rechte, substantielle Freiheiten und Chancen umfasst. Somit wird der gesellschaftspolitische Kontext als Opportunitätsstruktur für eine umfassende Teilhabe am gesellschaftlichen Leben konzeptionalisiert: Wohlfahrtsstaatliche, sozial- und arbeitsmarktpolitische Entscheidungen gestalten Lebens- und Partizipationschancen. Folgerichtig ist davon auszugehen, dass Lebenszufriedenheit als Ausweis von Lebensqualität in Abhängigkeit von wohlfahrtsstaatlichen Rahmenbedingungen variiert.

Das daraus abzuleitende Grundrecht auf ein gesichertes Existenzminimum ist auch unter Vertretern diverser Spielarten des Neoliberalismus unumstritten. Aber wie weit sollen staatliche Eingriffe und Balanceakte darüber hinausgehen, um welche Vorstellung von sozialer Gerechtigkeit zu verwirklichen? Die Soziologie betont die negativen Folgen sozialer Ungleichheit: Konflikte, mangelnde Systemunterstützung, Desintegration. In der wirtschaftspolitischen Diskussion findet sich hingegen die Behauptung, dass soziale Ungleichheit eine positive Funktion habe, weil sie den Wettbewerb fördere und Leistungsanreize setze. Gleichheit muss nicht als gerecht empfunden werden, wenn der Anspruch besteht, für unterschiedliche Leistung unterschiedlich entlohnt $\mathrm{zu}$ werden.

Die empirisch zu prüfende Frage lautet vor diesem Hintergrund: Inwiefern steht Gleichheit (im Sinne von Einkommensgleichheit) mit einer ausgeprägten Lebenszufriedenheit in Zusammenhang? Ist die Variation der wahrgenommenen Lebensqualität innerhalb Europas hinreichend erklärt, wenn man sich auf die Gleichverteilung von Einkommen konzentriert? Spielen nicht auch andere Qualitätsmerkmale einer Gesellschaft wie sozialer Schutz, Armutsvermeidung, Bildungszugang und das Erscheinungsbild einer Demokratie, die weniger für materielle Gleichheit als für Chancengleichheit, Sicherheit und Autonomie stehen, für das individuelle Wohlbefinden der Bevölkerung eine Rolle? 


\section{Individuelle und gesell- schaftliche Determinanten von Lebenszufriedenheit}

\subsection{EINKOMMEN UND INDIVIDUELLE LEBENSLAGEN}

Wie zufrieden jemand mit seinem Leben ist, hängt von vielen, miteinander verwobenen Faktoren ab. Das wissenschaftliche Interesse richtet sich darauf, biologische, psychologische und sozio-ökonomische Bedingungen in ihrer relativen Bedeutung und ihrem Zusammenspiel zu analysieren. Soziologische Untersuchungen zeigen beispielsweise, dass neben niedrigem Einkommen und einem unzureichenden Lebensstandard Krankheit, Einsamkeit und Arbeitslosigkeit mit geringer Lebenszufriedenheit in Verbindung stehen (Glatzer/Zapf 1984; Veenhoven 1984; Argyle 1999; Böhnke/Kohler 2010). Europaweit haben Personen mit höherem Einkommen eine höhere Lebenszufriedenheit als Personen mit niedrigem Einkommen. Eine Einkommenssteigerung geht mit Zufriedenheitszuwächsen einher. Wohlhabende Personen profitieren allerdings weniger von steigendem Einkommen, weil der zusätzliche Nutzen bei ohnehin schon hohem Einkommen geringer ausfällt als bei niedrigem. Der Zufriedenheitszuwachs ist darüber hinaus von begrenzter Dauer, weil Gewöhnungsprozesse eintreten, neue Begehrlichkeiten entstehen und man sich an anderen Bezugsgruppen ausrichtet (Helson 1964; Frederick/Loewenstein 1999). Neben dem absoluten Niveau des Lebensstandards zählt auch die individuelle Position im Vergleich zu anderen (Duesenberry 1949; Becker 1974; Easterlin 1974; Clark/ Oswald 1996; Firebaugh/Tach 2005).

\subsection{GESELLSCHAFTLICHE WOHLFAHRT}

Auch die ökonomische Prosperität des Landes ist bedeutsam für das subjektive Wohlbefinden: Je höher das Bruttoinlandsprodukt, desto höher die durchschnittliche Lebenszufriedenheit in einem Land (Inglehart/Klingemann 2000; Fahey/ Smyth 2004; Ryan/Deci 2001). Zwar findet sich das Phänomen des abnehmenden Grenznutzens auch auf Länderebene, und wegen des nichtlinearen Einkommensef- fektes hat ein steigendes Bruttoinlandsprodukt keine gleichermaßen steigende durchschnittliche Lebenszufriedenheit der Bevölkerung zur Folge (Easterlin 1973; Argyle 1999; Inglehart/Rabier 1986; Graham/Pettinato 2001; Hagerty/Veenhoven 2003; Blanchflower/Oswald 2001). Dennoch ist der Zusammenhang zwischen materiellem Wohlstand in einem Land und dem subjektiven Wohlbefinden der Bevölkerung eng.

Hinter diesem Zusammenhang verbergen sich allerdings komplexe Wirkungsmechanismen: Reiche Länder haben tendenziell stabilere Demokratien und weniger Korruption, verfügen über bessere soziale Standards und Versorgungssysteme und achten soziale Grund- und Menschenrechte. Folglich variiert die Lebenszufriedenheit im Ländervergleich nicht nur nach der Höhe des Bruttoinlandsprodukts, sondern auch nach dem Human Development Index und nach Indikatoren zum Erscheinungsbild und zur Leistungsfähigkeit einer Demokratie (Diener/Suh 1999; Inglehart/ Klingemann 2000; Diener/Lucas 2000; Helliwell 2003; Frey/Stutzer 2000 ), der Arbeitslosen- und Armutsquote sowie der Höhe der Arbeitslosenunterstützung und der Sozialschutzausgaben (Clark/Oswald 1994; Clark 2003; Di Tella et al. 2001).

Sozialstaatlicher Schutz stabilisiert in bedürftigen Lebenslagen nicht nur das Existenzminimum, sondern auch das Wohlbefinden. Sozialpolitische Rahmenbedingungen wie hohe Lohnersatzleistungen oder eine gute Gesundheitsfürsorge moderieren die Wirkung von Krankheit, Alter oder dem Ausscheiden aus dem Beruf auf die wahrgenommene Lebensqualität (Böhnke/Kohler 2007). Solche Wechselwirkungen zwischen individuellen Lebenslagen und staatlichen Sicherungssystemen zeigen sich auch anderweitig (Haller/Hadler 2006): Menschen, die wenig Vertrauen in die Sozialversicherungssysteme und in ihre Mitmenschen haben, die viele Konflikte zwischen gesellschaftlichen Gruppen wahrnehmen oder enttäuscht vom Gesundheits- und Bildungswesen ihres Landes sind, haben eine niedrigere Lebenszufriedenheit, und zwar unabhängig von ihrer individuellen Lebenslage (Böhnke 2008). Auch dies zeigt: Individuelle Lebensumstände erklären das Ausmaß an Lebenszufriedenheit nur zum Teil. Kulturelle, politische und ökonomische Rahmenbedingungen bestimmen die perzipierte Lebensqualität maßgeblich mit.
Vor diesem Hintergrund ist es verwunderlich, dass es keine ähnlich klaren Befunde für die Wirkung von Einkommensungleichheit auf das subjektive Wohlbefinden gibt. Einkommensungleichheit ist in Ländern mit einem hohen Sozialschutzniveau und ausgeprägten sozialpolitischen Programmen zur (Frauen-)Erwerbsintegration tendenziell geringer. Und doch ist der Zusammenhang zwischen Umverteilung und Einkommensungleichheit aufgrund unterschiedlicher Bevölkerungszusammensetzungen und differenziert wirksamen Marktmechanismen weniger ausgeprägt, als man gemeinhin annimmt (Esping-Andersen/Myles 2009).

Alesina et al. (2004) fanden heraus, dass sich - insbesondere arme - Europäer im Gegensatz zu US-Amerikanern von ungleich verteilten Einkommen in ihrem Land im Hinblick auf ihre Lebenszufriedenheit negativ beeinflussen lassen. Als Grund wird die höhere Akzeptanz und Opportunität sozialer Mobilität im angelsächsischen Raum benannt. Für intervenierende Mechanismen, die gegen einen direkten Zusammenhang zwischen Gleichheit und Lebenszufriedenheit sprechen, lassen sich auch Befunde der World Data Base of Happiness anführen: Skandinavische Länder mit einer geringeren Einkommensspreizung haben eine zufriedenere Bevölkerung als bspw. Länder in Südeuropa, wo die Einkommen sehr ungleich verteilt sind. Frankreich als ein Land mit relativ schwach ausgeprägter Einkommensungleichheit und gleichzeitig vergleichsweise niedriger durchschnittlicher Lebenszufriedenheit passt wiederum nicht in dieses Schema. Für die USA, Neuseeland und Großbritannien gilt ebenfalls ein anderer Zusammenhang: Hier ist die Lebenszufriedenheit trotz stark ungleich verteilter Einkommen hoch. Van Praag und Ferrer-I-Carbonell (2009) resümieren, dass Studien, die einen Zusammenhang zwischen hoher Einkommensungleichheit und niedriger Lebenszufriedenheit feststellen, die Prüfung anderer, sich dahinter verbergender Mechanismen vernachlässigen. Für Deutschland wiederum stellt jüngst eine Studie zum sogenannten Glücks-BIP fest, dass Einkommensungleichheit keinerlei Einbußen für die Lebenszufriedenheit nach sich ziehe (van Suntum et al. 2010).

Es ist also weder ausgemacht, noch ist der Gegenbeweis angetreten, dass Gleichheit, hier gemessen an der Einkommensverteilung in einem Land, der Gesamtbe- 
völkerung zu mehr Lebenszufriedenheit verhilft. Vielmehr legt der hier zusammengetragene Forschungsstand zwei wichtige Punkte nahe: Zum einen, dass subjektives Wohlbefinden als Indikator für Lebensqualität stark nach individuellen sozioökonomischen Lebenslagen differiert; zum anderen, dass es von gesamtgesellschaftlichen Strukturbedingungen abhängig ist, die zwar mit Chancengleichheit, Sicherheit und sozialer Gerechtigkeit zu umschreiben sind, aber nicht notwendigerweise die Gleichverteilung materieller Ressourcen bedeuten. Wie stellt sich dieser Zusammenhang empirisch dar?

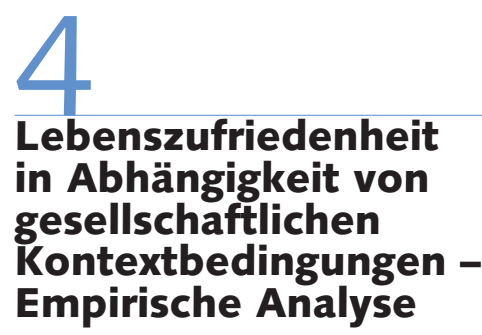

\subsection{INDIKATOREN}

Im Folgenden werden empirische Befunde gezeigt, die die durchschnittliche Lebenszufriedenheit in allen Ländern des erweiterten Europas in Beziehung zu gesellschaftlichen Kontextmerkmalen setzen, die für Gleichheit, Sicherheit und Autonomie stehen. Eine Operationalisierung dieser Konstrukte kann sich nur als Annäherung verstehen und ist selbstverständlich Kontroversen ausgesetzt. Ich werde in Anlehnung an Wilkinson/Pickett (2009) und die dadurch angestoßene Debatte Einkommensungleichheit als Indikator für das Ausmaß von Gleichheit in einer Gesellschaft verwenden.

Dabei kann man im Anschluss an die oben geführte Argumentation sicherlich nicht stehen bleiben. Als weitere Qualitätsmerkmale einer Gesellschaft greife ich deshalb auf die Ausgaben für Sozialschutz pro Kopf in Kaufkraftstandards zurück, auf die Höhe des Bruttoinlandsproduktes als Maß für absoluten Wohlstand sowie auf einen Indikator zur Effektivität und Steuerungsfähigkeit des politischen Systems (governance effectiveness) der Weltbank, der die Qualität der öffentlichen Dienste und die Glaubwürdigkeit der Regierung zu einer Maßeinheit verdichtet. Damit lässt sich annäherungsweise auf die Möglichkeiten für eine autonome Lebensführung schließen, wenn man darunter

\section{Abb. 1: Lebenszufriedenheit nach Einkommensgruppen in Europa 2007 \\ - sortiert nach dem Ausmaß an Einkommensungleichheit -}

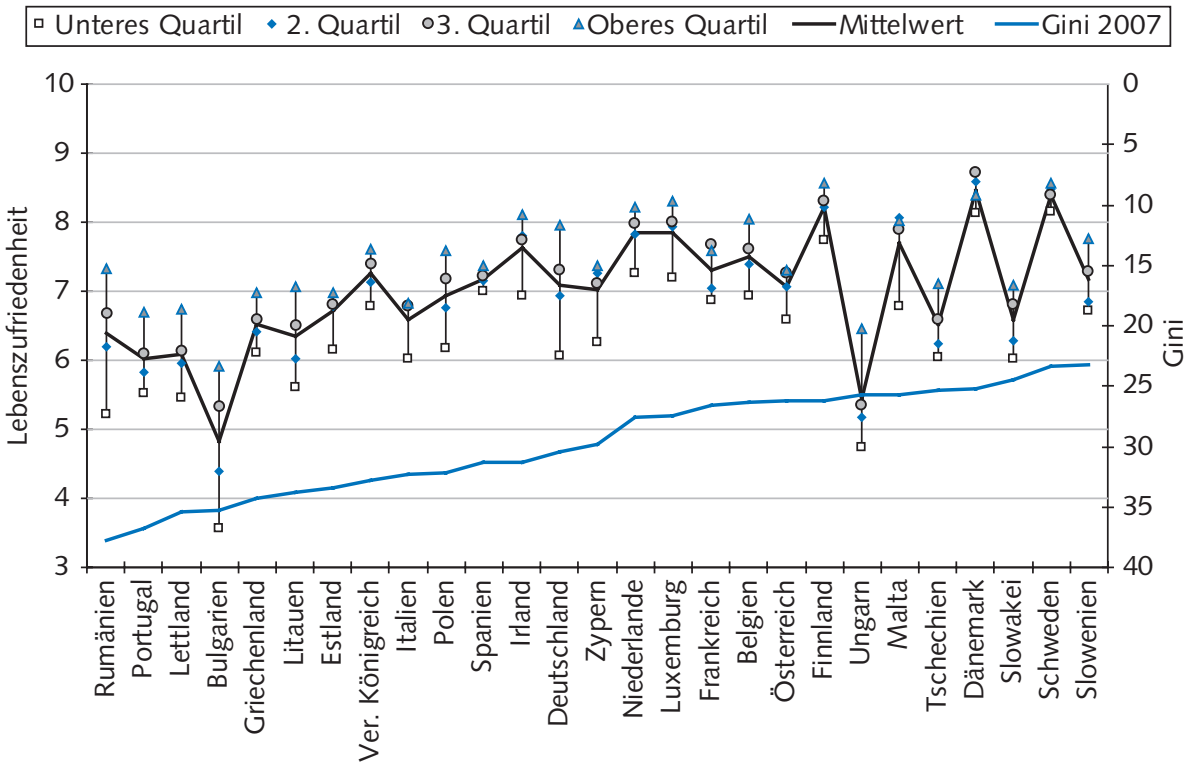

Quelle: Berechnungen der Autorin mit Daten des European Quality of Life Survey 2007. Gini-Index (nach Transferleistungen), hoher Wert=hohe Ungleichheit, Quelle: http://epp.eurostat.ec.europa.eu/-tgm/ table.do?tab=table\&init=1 \&language $=$ de\&pcode $=$ tessi190\&plugin $=0$, letzter Zugriff: 20.09 .2010

WSI MITTEILUNGEN

transparente institutionelle Strukturen und vertrauensvolle politische Rahmenbedingungen fassen möchte. Ähnlich zu verstehen ist ein weiterer Indikator, der die Durchlässigkeit des Bildungswesens illustriert. Hier werden Daten der Pisa-Studie 2006 herangezogen, die Lesekompetenz in Abhängigkeit vom sozio-ökonomischen Status darstellt. Ein Indikator zur Verringerung des Armutsrisikos durch Umverteilung rundet das Bild ab.

„All things considered, how satisfied would you say you are with your life these days?" Diese Frage liegt der Messung von Lebenszufriedenheit zugrunde. Die Antwortmöglichkeiten variieren von 1 (ganz und gar unzufrieden) bis 10 (ganz und gar zufrieden). Die Daten sind dem European Quality of Life Survey aus dem Jahr 2007 entnommen, der von der European Foundation for Living and Working Conditions im Auftrag der Europäischen Kommission durchgeführt wurde (Anderson et al. 2009).

\subsection{ERGEBNISSE}

Abbildung 1 zeigt alle 27 Länder der erweiterten EU geordnet nach dem Ausmaß an Einkommensungleichheit. Am linken Rand der Abbildung sehen wir Rumänien mit der am stärksten ausgeprägten Einkommensungleichheit, am rechten Rand Slowenien, das Land mit der geringsten Spreizung der Einkommen. Die durchgezogene schwarze Linie in der Grafik zeigt die durchschnittliche Lebenszufriedenheit in den jeweiligen Ländern. Wenn mit mehr Gleichheit bessere Lebensbedingungen und eine höhere Lebensqualität einhergingen, so müsste die Linie von links nach rechts ansteigend verlaufen. Tatsächlich trifft dies im Allgemeinen zu: Die durchschnittliche Lebenszufriedenheit ist in Ländern, in denen die Einkommensungleichheit geringer ausgeprägt ist, höher. Und doch weist die Lebenszufriedenheitskurve erhebliche Schwankungen auf. Einige ost- und mitteleuropäische Länder wie Ungarn, Tschechien und die Slowakei sind Ausreißer nach unten. Ihre Bevölkerung ist sehr viel unzufriedener, als man bei der vergleichsweise gering ausgeprägten Einkommensungleichheit vermuten sollte. Die skandinavischen Länder sind der Gegenpol mit einem sehr hohen allgemeinen Wohlbefinden der Einwohner. Dagegen wäre beispielsweise von Österreich - gemessen an der Ungleichverteilung ein höheres Niveau an Zufriedenheit zu erwarten. Dies sind plausible Hinweise darauf, dass nicht nur die Verteilung der 

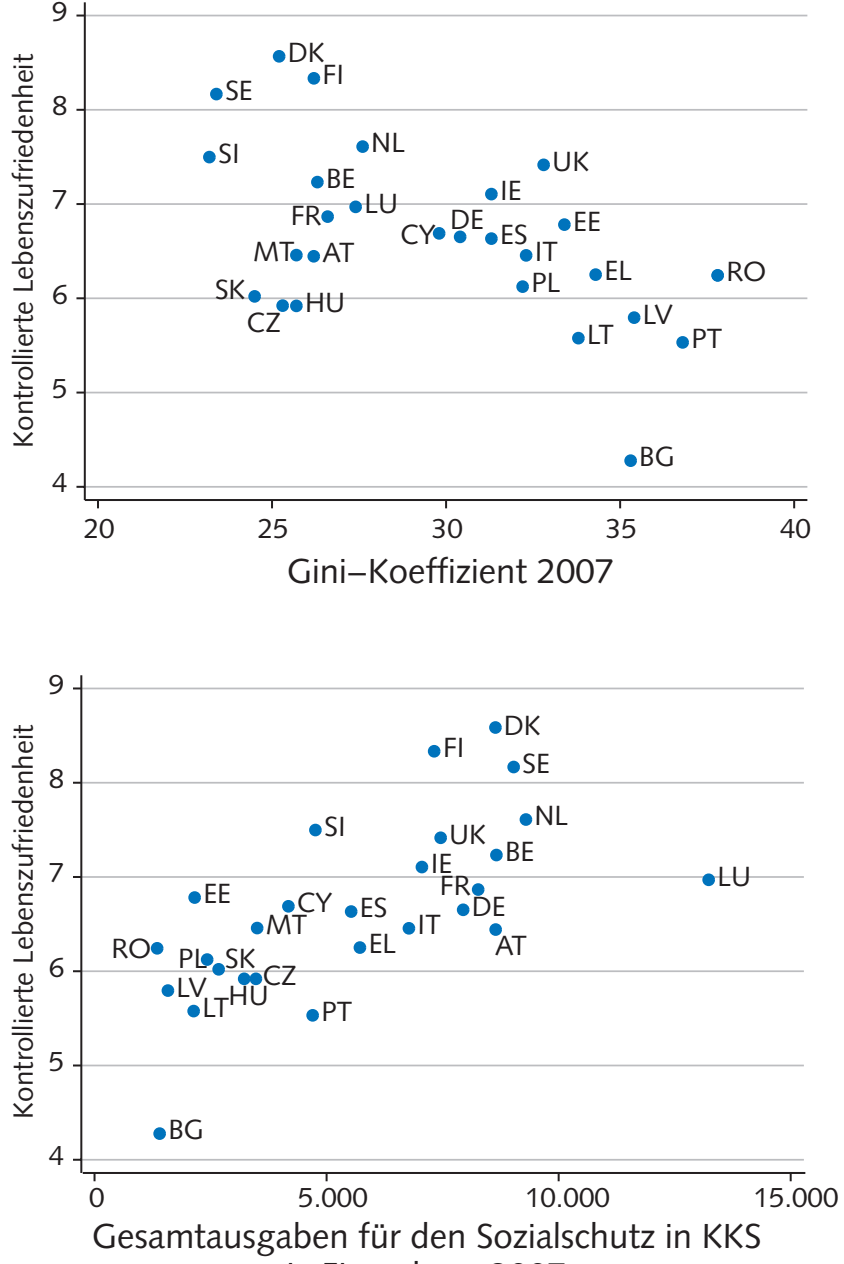

je Einwohner 2007

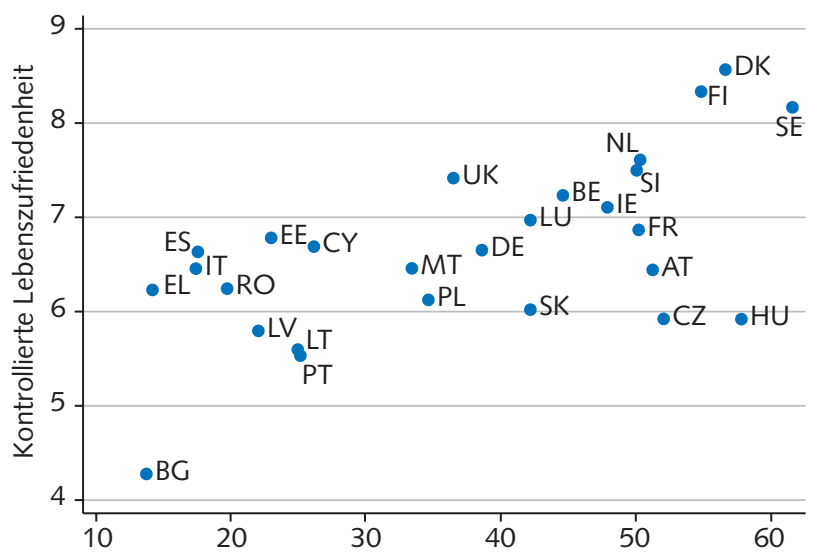

Bekämpfte Armut durch Umverteilung 2007 - in \% -
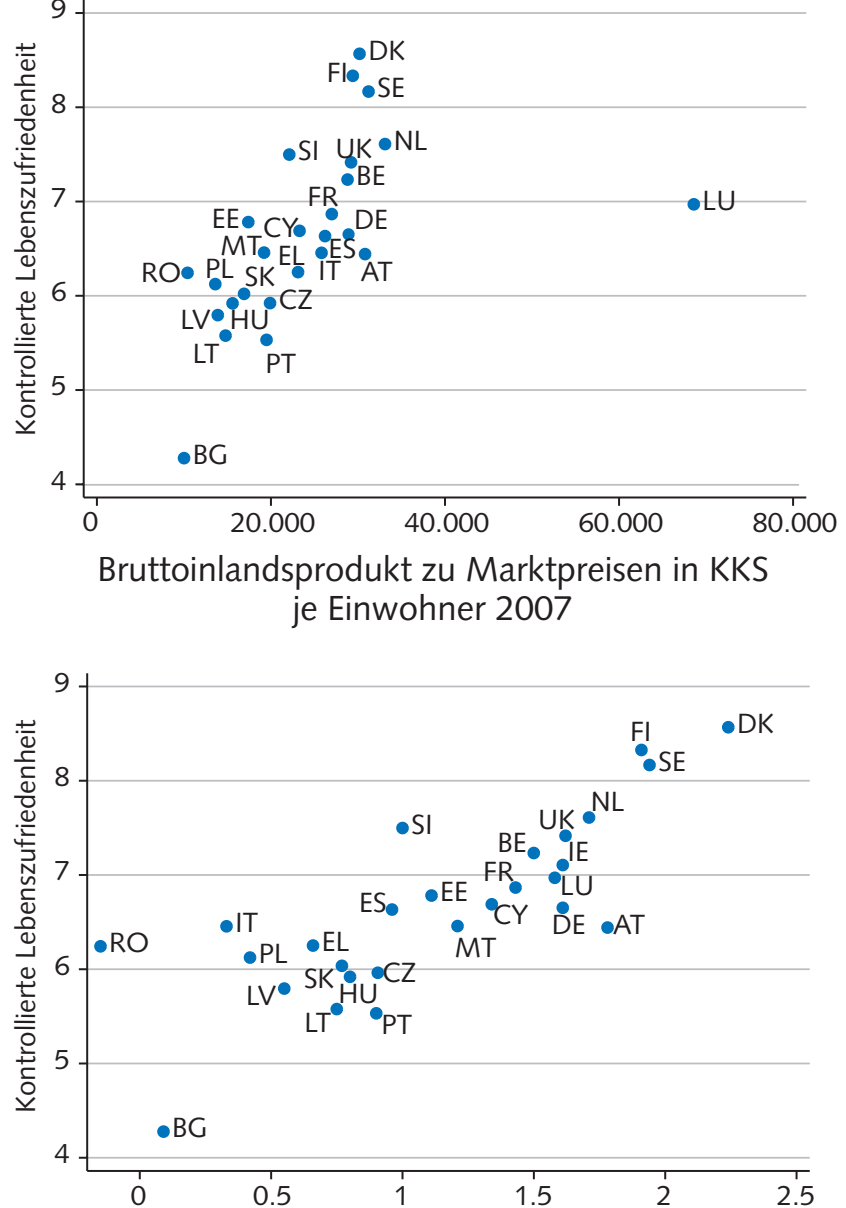

Steuerungswirksamkeit (Governance Effectiveness) 2007

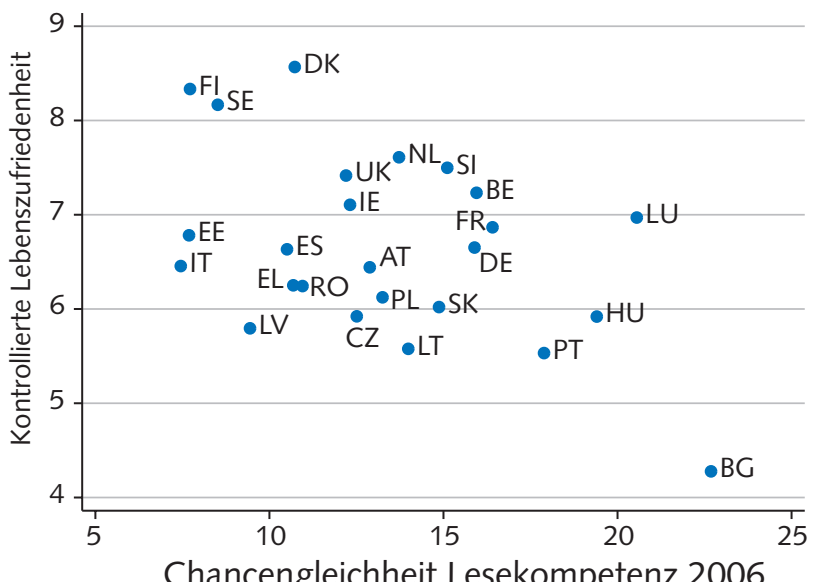

Chancengleichheit Lesekompetenz 2006

*Für Individualmerkmale kontrolliert, sodass Variationen zwischen den Ländern nicht auf Unterschiede in der Verteilung sozio-ökonomischer Merkmale zurückgeführt werden können.

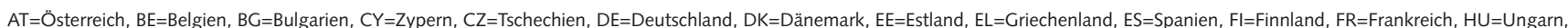

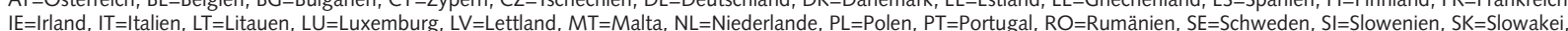
UE=Vereinigtes Königreich.

Quelle: Berechnungen und Darstellungen der Autorin mit Daten des European Quality of Life Survey 2007 plus Makroindikatoren: Gini-Index, siehe Abb. links oben; Bruttoinlandsprodukt zu Marktpreisen, Kaufkraftstandard je Einwohner, Quelle: http://epp.eurostat.ec.europa.eu/tgm/table.do?tab=table\&init1\&-language=de\&pcode=tec00001\&plugin=0, letzter Zugriff: 22.09.2010; Gesamtausgaben für Sozialschutz: beziehen sich auf Alter, Arbeitslosigkeit, Krankheit, Familie, Kinder, Wohnen etc., Quelle: http://epp.eurostat.ec.europa.eu/tgm/table.do?tab=table\&init=1 \&language=de\&pcode=tps00100\&plugin=0, letzter Zugriff: 29.09.2010; Governance Effectiveness, Quelle: http://info.worldbank.org/governance/wgi/mc_countries.asp, letzter Zugriff: 06.10.2010; Bekämpfte Armut durch Umverteilung: Armutsrisikoquote (60 \% des Median-Äquivalenzeinkommens) nach/vor Sozialleistungen multipliziert mit 100, Quelle: http://epp.eurostat.ec.europa.eu/ tgm/table.do?tab=table\&init=1\&language=de\&pcode=tesov250\&plugin=1, letzter Zugriff: 13.12.2010, http://epp.eurostat.ec.europa.eu/tgm/table.do?tab=table\&init=1\&language=de\&pcode =tsisc030\&plugin=0, letzter Zugriff: 13.12.2010; Chancenungleichheit Lesekompetenz: Varianzaufklärung der Lesekompetenz durch sozio-ökonomischen Status, je höher der Wert, desto stärker wird die Lekompetenz vom s0zio-ökonomischen Status dominiert, Quelle: http://www.pisa oecd org/datacd/30/18/39703566. pdf; Korrelationsstärken: Lebenszufriedenheit / Gini - 547: Lebenszufriedenheit / BIP 529 (ohne Luxemburg 747): Lebenszufriedenheit / Sozialschutz .657: Lebenszufriedenheit / Governance 788 ;

Lebenszufriedenheit / Bekämpfte Armut durch Umverteilung .613; Lebenszufriedenheit / Chancenungleichheit Lesekompetenz -.324 (ohne Malta und Zypern). 
Einkommen, sondern auch ihr absolutes Niveau eine Rolle für das subjektive Wohlbefinden spielen könnte, ebenso wie möglicherweise ein verlässliches Ausmaß an sozialem Schutz und Vertrauen in Institutionen.

Abbildung 1 weist darüber hinaus für jedes Land die Lebenszufriedenheit in Abhängigkeit von der Einkommenshöhe aus. Das Äquivalenzeinkommen ist in vier gleich große Gruppen aufgeteilt, wobei das unterste Quartil die niedrigsten Einkommen umfasst, dass obere Quartil die höchsten. Damit lassen sich mehrere wichtige Dinge gleichzeitig veranschaulichen. Wir sehen zum einen den Befund, dass die Lebenszufriedenheit in nahezu jedem Land deutlich nach Einkommensgruppen variiert. Bezieher hoher Einkommen sind mit ihrem Leben zufriedener als Bezieher niedriger Einkommen, dies ist ein länderübergreifend gültiges Muster. Die Unterschiede zwischen den einzelnen Einkommensgruppen sind allerdings nicht immer gleich groß. Hier lässt sich bei grober Deutung der Trend ablesen, dass in Ländern mit hoher Einkommensungleichheit auch die Ungleichheit in der Verteilung der Lebenszufriedenheit groß ist. Erneut tragen die osteuropäischen Länder diesen Trend aber häufig nicht mit. Auch südeuropäische Länder weisen trotz relativ stark ausgeprägter Einkommensungleichheit keine große Spreizung beim allgemeinen Wohlbefinden auf. Dies sind wiederum Hinweise auf komplexere Wirkungsmechanismen, die den vermeintlich eindeutigen und direkten Zusammenhang zwischen Gleichheit und Lebensqualität infrage stellen.

Wir finden damit Forschungsergebnisse reproduziert, die die hohe Relevanz individueller sozio-ökonomischer Lebensbedingungen für die allgemeine Lebenszufriedenheit betonen und letztlich unumstritten sind: Das verfügbare Einkommen als Platzhalter für materielle Ressourcen und Lebensstandard bestimmt neben Bildung, Erwerbstätigkeit, Gesundheit und der Einbindung in soziale Netzwerke maßgeblich das individuelle Wohlbefinden in jedem europäischen Land. Damit sind aber die Länderunterschiede in der allgemeinen Lebenszufriedenheit nicht hinreichend erklärt, und die Bedeutsamkeit von gesellschaftlichen Kontextmerkmalen, die es Individuen ermöglichen, bestimmte Lebensbedingungen zu realisieren, rückt in den Mittelpunkt.
Um auf die zusätzliche Bedeutung von beispielsweise absolutem Wohlstand, Einkommensungleichheit, Sozialschutz und der Funktionsweise einer Demokratie schließen zu können, braucht es komplexere Berechnungsmethoden. Im Folgenden wird eine vereinfachte Darstellung gewählt, um die Ergebnisse von Mehrebenenmodellen anschaulich visualisieren zu können. Abbildung 2 zeigt den Einfluss der Makroindikatoren auf eine hinsichtlich bestimmter individueller Merkmale kontrollierte Lebenszufriedenheit. Damit wird eine Lebenszufriedenheit abgebildet, die bereits um den Einfluss von Alter, Geschlecht, Bildung, Einkommen, Familienstand und Erwerbsstatus bereinigt ist. Dies bedeutet: Variationen der Lebenszufriedenheit zwischen den Ländern können nicht mehr auf Unterschiede in der Verteilung dieser sozio-ökonomischen Merkmale in der jeweiligen Bevölkerung zurückgeführt werden, sondern zeigen ausschließlich auf, wie eng sie mit den verschiedenen, in Abbildung 2 abgetragenen gesellschaftlichen Rahmenbedingungen, zusammenhängen.

Die Berechnungen ergeben, dass die Makroindikatoren einen im statistischen Sinne signifikanten Einfluss auf die Lebenszufriedenheit ausüben, unabhängig von ihrer Interaktion mit sozio-ökonomischen Charakteristika der Bevölkerung eines Landes. So erklären zwar Merkmale einer Person, wie bspw. Arbeitslosigkeit, Armut und Alter, im Schnitt in Europa etwa $14 \%$ der Varianz der Lebenszufriedenheit. Die gesellschaftlichen Kontextmerkmale können aber davon unabhängig weitere $9 \%$ hinzufügen. Mit anderen Worten: Die wahrgenommene Lebensqualität wird zu einem nicht unerheblichen Teil von gesellschaftlichen Rahmenbedingungen wie Gleichheit, Sicherheit, Wohlstand und Autonomie geprägt. Unabhängig vom individuellen Bildungsniveau, von der eigenen Arbeitsmarktanbindung oder dem eigenen Einkommen beeinflussen auch der allgemeine Wohlstand, die Chancengleichheit und das Niveau sozialer Sicherung in einem Land die Lebenszufriedenheit einer Person in einem relevanten Ausmaß.

Abbildung 2 zeigt diese Zusammenhänge plastisch auf: Die für sozio-ökonomische Individualmerkmale kontrollierte Lebenszufriedenheit variiert in Abhängigkeit der ausgewiesenen gesellschaftlichen Kontextbedingungen. Wir sehen: Die Lebenszufriedenheit ist tendenziell höher in Ländern mit einem höheren absoluten Wohlstandsniveau gemessen am Bruttosozialprodukt, ebenso dort, wo die Ausgaben für sozialen Schutz generöser sind, Armut effizienter bekämpft wird, Chancengleichheit im Bildungswesen eher verwirklicht ist, die Einkommen näher beieinander liegen und die Qualität der Regierung und öffentlichen Dienste besser ist. Schließlich ist tendenziell dort, wo die Lesekompetenz stark von sozioökonomischen Merkmalen abhängt - die Chancengleichheit in diesem Punkt also gering ist -, auch die Lebenszufriedenheit geringer. Dieser Zusammenhang ist allerdings relativ schwach ausgeprägt.

Der Zusammenhang zwischen Einkommensungleichheit und Lebenszufriedenheit ist durchaus vorhanden, aber vergleichsweise gering, das zeigen auch die entsprechenden Korrelationskoeffizienten aus den Modellrechnungen. Dazu passt die Tatsache, dass die Makroindikatoren zwar alle miteinander korrelieren, denn Länder mit hohem Bruttosozialprodukt sind tendenziell auch eher die Länder, die mehr für sozialen Schutz ausgeben und effektiver regieren. In Nordeuropa finden wir Länder mit ausgeprägtem Sozialschutz, gleichzeitig hoher Besteuerung und entsprechend geringerer Einkommensspreizung - die Bevölkerung dieser Länder ist am zufriedensten. Einkommensungleichheit folgt aber nicht immer diesem Schema: Eine Reihe von Ländern mit relativ gleich verteilten Einkommen ist schwächer in anderen Bereichen, die für die Qualität einer Gesellschaft auch ausschlaggebend sind. Hier lässt sich nur schwer ein Muster erkennen. Dies ist ein weiterer Hinweis darauf, dass Gleichheit (zumindest in Form von Einkommensungleichheit) als Einflussfaktor für Lebensqualität möglicherweise erst ausschlaggebend wird, wenn ein bestimmtes Lebensstandard-, Vertrauens- und Schutzniveau über existenzielle Bedürfnisse hinaus gegeben ist. Dafür spricht der vergleichsweise enge Zusammenhang zwischen Lebenszufriedenheit, Sozialschutz und Armutsbekämpfung.

\section{Resümee}

Die Lebenszufriedenheit eines Menschen spiegelt wider, wie gut seine materielle und immaterielle Lebens- und Versorgungssituation ist. Sie lässt gleichermaßen auf 
Einkommen, Wohnbedingungen und Teilhabechancen schließen und hängt von der Position am Arbeitsmarkt ebenso ab wie vom Gesundheitszustand. Aber dieses zusammenfassende Maß wahrgenommener Lebensqualität verweist auch auf etwas, was darüber hinausgeht: Auf die ökonomischen, institutionellen und gesellschaftspolitischen Rahmenbedingungen eines Landes, die Lebensverläufe und Lebenschancen maßgeblich prägen.

Die durchschnittliche Lebenszufriedenheit variiert zwischen den Ländern Europas erheblich. Ausgehend von der populären These, dass die Gleichverteilung der Einkommen - und nicht etwa das generelle Lebensstandardniveau - in einem Land die Lebensqualität seiner Bewohner maßgeblich beeinflusst, haben wir gefragt, welchen Stellenwert gesellschaftliche Rahmenbedingungen unterschiedlicher Art, die auf ein bestimmtes wohlfahrtsstaatliches Strukturgefüge eines Landes schließen lassen, für die Lebenszufriedenheit haben. Dem Forschungsstand und eigenen Berechnungen können wir folgende Tendenzen entnehmen: Je höher die Sozialschutzausgaben, je effektiver die Armutsbekämpfung und das politische System und je höher die allgemeine wirtschaftliche Prosperität in einem Land, desto zufriedener sind die Menschen mit ihrem Leben. Wird sozialer Schutz als prekär empfunden und mangelt es an Vertrauen in die öffentliche Dienste und die Regierung, sehen Menschen ihre persönlichen Lebensumstände davon beeinträchtigt. Weitere Kontextfaktoren wie die Arbeitslosenquote, der ungleiche Zugang zum Bildungswesen, Korruption und Säuglingssterblichkeit sind relevant und lassen die Lebenszufriedenheit sinken. Die Menschen sind in der Lage, die Qualität ihrer Gesellschaft und ihrer einzelnen Systeme einzuschätzen und nehmen sie als Gestaltungsspielraum oder Restriktion für ihre eigenen Lebenschancen wahr.

Lebenszufriedenheit als subjektive Bilanz von Lebensqualität bezieht sich auf ein ganzes Bündel von Faktoren. Sie reflektiert nicht nur das absolute Lebensstandardniveau und die relative Position eines Individuums im Wohlstandsgefüge des eigenen Landes, sondern ist gleichzeitig Gradmesser für die Qualität der politischen und kulturellen Rahmenbedingungen eines Landes. Dabei spielt Gleichheit im Sinne einer Gleichverteilung materieller Ressourcen zwar eine wichtige Rolle, trägt aber zur Erklärung der Variation von Lebens- zufriedenheit im Ländervergleich nicht in der dominanten Art und Weise bei, wie gegenwärtig oft unterstellt wird. Die Gleichverteilung von Einkommen nützt den Menschen wenig, wenn sie auf einem so niedrigen Niveau gleich verteilt sind, dass existenzielle Schutz- und Sicherheitsbedürfnisse nicht befriedigt werden können. Stattdessen geht es um ein ganzes Bündel institutioneller Strukturen. Dieses umfasst das Ausmaß an (Chancen-) Gleichheit, die Sicherung eines Existenzminimums und den Schutz bei Lebensrisiken wie Krankheit und Erwerbslosigkeit und bezieht sich darüber hinaus auch auf die Verlässlichkeit und Vertrauenswürdigkeit politischer Akteure, Institutionen und Systeme. Diese gesellschaftlichen Rahmenbedingungen können als Opportunitätsstruktur für Lebensqualität verstanden werden, die sozialer Gerechtigkeit förderlich sind und Chancengleichheit verbessern. Der Anspruch an ein selbstbestimmtes Leben ist nicht einzulösen, wenn es an wohlfahrtsstaatlichen Prinzipien entsprechenden Schutzund Ausgleichsmechanismen fehlt. Eine Verengung der Diskussion auf den Aspekt materieller Gleichheit vernachlässigt die Komplexität dessen, was Lebensqualität ausmacht.

\section{LITERATUR}

Alesina, A./Di Tella, R./MacCulloch, R. (2004): Inequality and Happiness: Are Europeans and Americans Different?, in: Journal of Public Economics 88 (9-10), S. 2009-2042

Allardt, E. (1976): Dimensions of Welfare in a Comparative Scandinavian Study, in: Acta Sociologica 19 (3), S. 227-239

Allardt, E. (1993): Having, Loving, Being: An Alternative to the Swedish Model of Welfare Research, in: Nussbaum, M. /Sen, A. (Hrsg.): The Quality of Life, Oxford, S. 88-94

Anderson, R./Mikuliç, B./Vermeylen G./Lyly-Yrjanainen M./Zigante, V.; European Foundation for the Improvement of Living and Working Conditions (Hrsg.) (2009): Second European Quality of Life Survey - Overview, Office for Official Publications of the European Communities, Luxembourg

Argyle, M. (1999): Causes and Correlates of Happiness, in: Kahnemann, D./Diener, E./Schwarz, N. (Hrsg.): Well-Being: The Foundations of Hedonic Psychology, New York, S. 353-373

Becker, G. (1974): A Theory of Social Interactions, in: Journal of Political Economy 82 (6), S. 1063-1093
Blanchflower, D. G./Oswald, A. J. (2001): Unemployment, Well-being and Wage Curves in Eastern Europe, in: Journal of the Japanese and International Economies 15 (4), S. 364-402

Böhnke, P. (2008): Does Society Matter? Life Satisfaction in the Enlarged Europe, in: Social Indicators Research 87 (2), S. 189-210

Böhnke, P./Kohler, U. (2007): Determinanten des Glücks: Lebenszufriedenheit in Europa, in: WSI-Mitteilungen (60) 7, S. 373-379

Böhnke, P./Kohler, U. (2010): Well-Being and Inequality, in: Immerfall, St./Therborn, G. (Hrsg.): Handbook of European Societies, Heidelberg, S. 629-666

Campbell, A./Converse, P. E./Rodgers, W. L. (1976): The Quality of American Life. Perceptions, Evaluations, and Satisfactions, New York Clark, A. E. (2003): Unemployment as a Social Norm: Psychological Evidence from Panel Data, in: Journal of Labor Economics 21 (2), S. 323-351

Clark, A. E./Oswald, A. J. (1994): Unhapppiness and Unemployment, in: Economic Journal 104 (424), S. 648-659 
Clark, A. E./Oswald, A. J. (1996): Satisfaction and Comparison Income, in: Journal of Public Economics 61 (3), S. 359-381

Dahrendorf, R. (1992): Der moderne soziale Konflikt: Essay zur Politik der Freiheit, Stuttgart

Di Tella, R./MacCulloch, R. J./Oswald, A. J. (2001): Preferences over Inflation and Unemployment: Evidence from Surveys of Happiness, in: American Economic Review 91 (1), S. 335-341

Diener, E./Suh, E. M. (1999): National Differences in Subjective WellBeing, in: Kahneman, D./Diener, E./Schwarz, N. (Hrsg.): Well-Being: The Foundations of Hedonic Psychology, New York, S. 434-450

Diener, E./Suh, E. M./Lucas, R./Smith, H. (1999): Subjective WellBeing: Three Decades of Progress, in: Psychological Bulletin 125 (2), S. 276-302

Diener, E./Lucas R. (2000): Explaining Differences in Societal Levels of Happiness: Relative Standards, Need Fulfillment, Culture, and Evaluation Theory, in: Journal of Happiness Studies: An Interdisciplinary Periodical on Subjective Well-Being 1 (1), S. 41-78

Duesenberry, J. S. (1949): Income, Savings and the Theory of Consumer Behavior, Cambridge, Mass.

Easterlin, R. A. (1973): Does Money Buy Happiness?, in: The Public Interest 30, S. 3-10

Easterlin, R.A. (1974): Does Economic Growth Improve the Human Lot? Some Empirical Evidence, in: David, P. A./Reder, M. W. (Hrsg.): Nations and Households in Economic Growth: Essays in Honour of Moses Abramowitz, New York/London, S. 89-125

Erikson, R. (1974): Welfare as a Planning Goal, in: Acta Sociologica 17 (3), S. 273-278

Esping-Andersen, G./Myles, J. (2009): Economic Inequality and the Welfare State, in: Salverda, W./Nolan, B./Smeeding, T.M. (Hrsg.): The Oxford Handbook of Economic Inequality, Oxford, S. 639-664

Fahey, T./Smyth, E. (2004): Do Subjective Indicators Measure Welfare? Evidence from 33 European Societies, in: European Societies 6 (1), S. 5-27

Firebaugh, G./Tach, L. (2005): Relative Income and Happiness: Are Americans on a Hedonic Treadmill?, Working Paper, hrsg. von der Pennsylvania State University, Pennsylvania

Frederick, S./Loewenstein, G. (1999): Hedonic Adaptation, in: Kahnemann, D./Diener, E./Schwarz, N.(Hrsg.): Well-Being: The Foundations of Hedonic Psychology, New York, S. 302-329

Frey, B. S./Stutzer, A. (2000): Happiness, Economy and Institutions, in: Economic Journal 110 (446), S. 918-938

Glatzer, W./Zapf, W. (1984): Lebensqualität in der Bundesrepublik. Objektive Lebensbedingungen und subjektives Wohlbefinden, Frankfurt/New York

Graham, C./Pettinato, S. (2001): Happiness, Markets and Democracy: Latin America in Comparative Perspective, in: Journal of Happiness Studies 2 (3), S. 237-268

Hagerty, M./Veenhoven, R. (2003): Wealth and Happiness Revisited - Growing National Income Does Go with Greater Happiness, in: Social Indicators Research 64 (1), S. 1-27
Haller, M./Hadler, M. (2006): How Social Relations and Structures Can Produce Happiness and Unhappiness: An International Comparative Analysis, in: Social Indicators Research 75 (2), S. 169-216

Helliwell, J. F. (2003): How's Life? Combining Individual and National Variables to Explain Subjective Well-being, in: Economic Modelling 20 (2), S. 331-360

Helson, H. (1964): Adaptation-Level Theory: An Experimental and Systematic Approach to Behavior, New York Inglehart, R./Rabier, J.-R. (1986): Aspirations Adapt to Situations But Why Are the Belgians so Much Happier than the French?, in: Andrews, F. M. (Hrsg.): Research on the Quality of Life, Ann Arbor, S. 1-56

Inglehart, R./Klingemann, H.-D. (2000): Genes, Culture, Democracy, and Happiness, in: Diener, E./Suh, E. M. (Hrsg.): Culture and Subjective Well-being, Cambrige, Mass., S. 165-183

Layard, R. (2005): Happiness. Lessons from a New Science, Harmondsworth

Liebig, St. (2010): „Gleichheit ist Glück. Warum gerechte Gesellschaften für alle besser sind " Oder: Einkommensgleichheit als Lösung aller gesellschaftlichen Probleme?, in: Gesellschaft - Wirtschaft - Politik (GWP) 59 (3), S. 407-413

Marshall, T. H. (1992 [1949]): Bürgerrechte und soziale Klassen. Zur Soziologie des Wohlfahrtsstaates, Frankfurt/New York

Ryan, M. R./Deci, A. L. (2001): On Happiness and Human Potentials: A Review of Research on Hedonic and Eudaimonic Well-being, in: Annual Review of Psychology 52, S. 141-66

Saunders, P. (2010): Beware False Prophets. Equality, the Good Society and The Spirit Level, Policy Exchange, London

Sen, A. (1999): Development as Freedom, Oxford

Stiglitz, J./Sen, A./Fitoussi J.-P. (2009): Report of the Commission on the Measurement of Economic Performance and Social Progress, http://www.stiglitz-sen-fitoussi.fr

Van Praag, B./Ferrer-I-Carbonell, A. (2009): Inequality and Happiness, in: Salverda, W./Nolan, B./Smeeding, T. (Hrsg.): The Oxford Handbook of Economic Inequality, Oxford, S. 364-383

Van Suntum, U./Prinz, A./Uhde, N. (2010): Lebenszufriedenheit und Wohlbefinden in Deutschland: Studie zur Konstruktion eines Lebenszufriedenheitsindikators, SOEPpapers on Multidisciplinary Panel Data Research at DIW Berlin (259), Berlin

Veenhoven, R. (1984): Conditions of Happiness, Dordrecht Wilkinson, R./Pickett, K. (2009): The Spirit Level. Why Equality Is Better for Everyone, London

Zapf, W. (1972): Zur Messung der Lebensqualität, in: Zeitschrift für Soziologie 1(4), S. 353-376

Zapf, W. (1999): Qualität des Lebens - Wege und Irrwege einer langen Entwicklung, in: Lehner, F. (Hrsg.): WertSchöpfung. Maßstäbe einer neuen Ökonomie, München/Mering, S. 162-170 